\title{
miR-10b promotes invasion by targeting HOXD10 in colorectal cancer
}

\author{
YUNFENG WANG ${ }^{1}$, ZHEN LI $^{1}$, XUHONG ZHAO ${ }^{2}$, XIAOMING ZUO $^{3}$ and ZHIHAI PENG ${ }^{4}$ \\ Departments of ${ }^{1}$ General Surgery, ${ }^{2}$ Central Experiment Laboratory and ${ }^{3}$ Pathology, \\ Yangpu Hospital, Tongji University School of Medicine, Shanghai 200090; ${ }^{4}$ Department of General Surgery, \\ Shanghai First People's Hospital, School of Medicine, Shanghai Jiao Tong University, Shanghai 200030, P.R. China
}

Received March 19, 2015; Accepted April 8, 2016

DOI: $10.3892 / \mathrm{ol} .2016 .4628$

\begin{abstract}
Studies have shown that homeobox D10 (HOXD10) is the target gene of microRNA-10b (miR-10b) and is closely associated with the inhibition of cell migration and invasion. Ras homolog family member C (RhoC) has been reported to promote tumor metastasis in various types of cancer. The effect of miR-10b on colorectal cancer (CRC) metastasis and the associated molecular mechanisms remain elusive. The present study aimed to investigate whether miR-10b could promote invasion by targeting HOXD10 in CRC by exploring the association between miR-10b and HOXD10 expression in $\mathrm{CRC}$ patients. The findings revealed that miR-10b levels were elevated in the CRC specimens and significantly correlated with advanced clinical stage and lymph node metastasis. In addition, HOXD10 was a direct target of miR-10b, and the increased expression of RhoC and downregulation of HOXD10 correlated with the increased expression level of miR-10b. HOXD10 protein level was also markedly attenuated in lymph node metastasis-positive tumor tissues compared with lymph node metastasis-free tumor tissues. These findings suggest that miR-10b may stimulate the upregulation of RhoC through targeting HOXD10, thus promoting the invasion and migration in $\mathrm{CRC}$ tumor.
\end{abstract}

\section{Introduction}

With the rapid development of the worldwide economy, the structure of people's diets has changed in recent years (1). Colorectal cancer (CRC) has become one of the most common malignant tumors of the digestive tract in China, and is the major cause of gastrointestinal cancer-associated mortality (2). One reason for the high frequency of tumor

Correspondence to: Dr Yunfeng Wang, Department of General Surgery, Yangpu Hospital, Tongji University School of Medicine, 450 Teng Yue Road, Shanghai 200090, P.R. China

E-mail: wangyunfeng197911@163.com

Key words: colorectal cancer, microRNA-10b, homeobox D10, migration metastasis is that the majority of CRC patients are diagnosed at an advanced stage (3). Malignant CRCs often possess the characteristics of rapid progression and invasion, which contribute to local and liver metastases and can result in a poor prognosis (4,5). MicroRNAs (miRs) are known to be involved in a variety of physiological processes, including cell survival, growth and metabolism (6). Studies have demonstrated that miRs are involved in the regulation of metastasis in various types of cancer $(7,8)$. Several types of $\mathrm{miR}$ have been found to be important for the process of tumor metastasis, suggesting that miRs may be used as a potential transfer marker $(9,10)$.

The twist-induced miR, miR-10b, has been previously found to promote tumor metastasis in cancer (11). One study indicated that homeobox D10 (HOXD10) is the target gene of miR-10b and is closely associated with the inhibition of cell migration and invasion (12). The expression level of HOXD10 was found to decrease during the malignant progression of cancer (13). The ras homolog family member C (RhoC) protein is a member of the Ras superfamily of guanine triphosphate (GTP)-binding proteins. Numerous studies have indicated that tumor metastasis is associated with an abnormal AKT signaling pathway, as AKT mediates a variety of basic cellular processes, including the resistance of cells to apoptosis and cell cycle effects (14). Although certain studies have reported a potential association between miR-10b and CRC $(15,16)$, the effect of miR-10b on CRC metastasis and the associated molecular mechanism remain elusive.

The purpose of the present study was to investigate the expression of miR-10b in CRC patients, to evaluate the effect of miR-10b on CRC invasion and migration and to determine whether miR-10b expression is mediated by HOXD10 targeting RhoC. The results of the present study showed that the overexpression of miR-10b in patients with CRC was associated with a decreased level of HOXD10 protein, which was accompanied by the increased expression of RhoC. These results suggested that miR-10b may be important in the migration and invasion of CRC.

\section{Materials and methods}

Clinical summary. A total of 126 primary CRC samples were collected consecutively during surgery from patients with 
histologically confirmed CRC, who underwent surgical resections at the Department of General Surgery, Yangpu Hospital, Tongji University School of Medicine (Shanghai, China) between January 2012 and January 2014. Adjacent healthy tissues were also taken from these patients with CRC to form the adjacent non-tumor tissue group. Additionally, 126 healthy people, who had undergone physical examinations in the same hospital, were used as a control group. Tissue Specimens were obtained from colonoscopy, and were immediately frozen in liquid nitrogen and stored at $-80^{\circ} \mathrm{C}$ until further processing. Only CRC patients that had not received any preoperative radio or chemotherapy were enrolled in the present study. All patients were staged according to the International Union Against Cancer of CRC TNM Staging system (2010; 7th edition) for CRC (17). The present study was conducted in accordance with the Declaration of Helsinki (18) and with approval from the Ethics Committee of Yangpu Hospital, Tongji University School of Medicine. Written informed consent was obtained from all participants.

Sample treatment. Adjacent non-cancerous tissues were carefully dissected from the surrounding benign tissues by a certified pathologist, and the margin was confirmed through immunohistochemistry by two independent pathologists at the Department of Pathology, Yangpu Hospital, Tongji University School of Medicine. The surrounding non-tumor tissues were used as control for each tumor tissue. All samples were immediately frozen in liquid nitrogen following surgical removal until required.

RNA extraction and reverse transcription $(R T)$-quantitative polymerase chain reaction $(q P C R)$. Total RNA was extracted from the resected tissues for the messenger RNA (mRNA) expression assay using TRIzol ${ }^{\circledR}$ reagent (Gibco; Thermo Fisher Scientific, Inc. Waltham, MA, USA). Lysed samples were bound to a silica-based filter and treated with DNase I (20,000 units; Invitrogen; Thermo Fisher Scientific, Inc.) RT-PCR was performed using the Reverse Transcriptase kit (Thermo Fisher Scientific, Inc.), according to the manufacturer's instructions. After complementary DNA was obtained, RT-PCR was performed using the SYBR Green PCR reagent kit (Thermo Fisher Scientific, Inc.). qPCR was performed using an initial denaturation step at $95^{\circ} \mathrm{C}$ for $5 \mathrm{~min}$, then 40 cycles of amplification, consisting of denaturation at $95^{\circ} \mathrm{C}$ for $15 \mathrm{sec}$, annealing at $60^{\circ} \mathrm{C}$ for $30 \mathrm{sec}$ and elongation at $72^{\circ} \mathrm{C}$ for $30 \mathrm{sec}$ on a 7500 Fast Real-time PCR System (Applied Biosystems; Thermo Fisher Scientific, Inc.). All 20- $\mu 1$ reactions were performed in triplicate. 5S ribosomal RNA (rRNA) was used as the internal control. The primer sequences were as follows: miR-10b, forward, 5'-TACCCTGTAGAACCGAATTTG-3' and reverse, 5'-AAC TGGTGTCGTGGAGTCGGC-3'; primers used for detection of HOXD10, forward, 5'-GACATGGGGACCTATGGAATG C-3' and reverse, 5'-TGGTGGTTCACTTCTCTTTTGG-3'; and 5S rRNA, forward, 5'-CCATACCACCTGGAAACGC-3' and reverse, 5'-TACTAACCGAGCCCGACCCT-3' (Jrdun Biotechnology, Shanghai, China). Data analysis for miR expression was performed using the $2^{-\Delta \Delta \mathrm{Cq}}$ method (19).

Western blot analysis. Cold radioimmunoprecipitation assay lysis buffer (Beyotime Institute of Biotechnology, Haimen, China) was used to extract proteins from solubilized tissues.
Protein assay reagents (Bio-Rad Laboratories, Inc., Hercules, CA, USA) were used to determine the protein concentration. Equal amounts of protein was then separated using a NuPAGE 4-12\% Bis-Tris gradient gel (Invitrogen; Thermo Fisher Scientific, Inc.) and transferred to a polyvinylidene fluoride membrane. The membrane was incubated with primary polyclonal goat RhoC (dilution, 1:200; catalog no., sc-12116), polyclonal goat HOXD10 (dilution, 1:200; catalog no., sc-33004) and polyclonal goat $\beta$-actin (dilution, 1:10,000; catalog no., sc-1616) (Santa Cruz Biotechnology, Inc., Dallas, TX, USA) antibodies overnight at $4^{\circ} \mathrm{C}$, blocked with $2 \%$ non-fat milk in phosphate-buffered saline (PBS)/Tween-20, and then incubated at room temperature with $5 \mathrm{mg}$ anti-goat $\mathrm{IgG}$ horseradish peroxidase (HRP)-conjugated antibodies (dilution, 1:10,000; catalog no., AS09605; Santa Cruz Biotechnology, Inc.) for $45 \mathrm{~min}$. An enhanced chemiluminescence reaction (Invitrogen; Thermo Fisher Scientific, Inc.) was performed for $40 \mathrm{~min}$. The relative densities of proteins was quantified with Image J software v.1.48u (National Institutes of Health, Bethesda, MD, USA).

Immunohistochemistry. Tumor or matched normal tissues were fixed in $4 \%$ paraformaldehyde, paraffin embedded, and sliced into $4 \mu \mathrm{m}$ sections. The primary antibody used for HOXD10 immunostaining was a mouse monoclonal anti-HOXD10 antibody (dilution, 1:50; Santa Cruz Biotechnology, Inc.). The sections were incubated with $5 \mathrm{mg}$ HRP-conjugated anti-mouse IgG polyclonal secondary antibody (dilution, 1:10,000; catalog no., NB910-95603; Abcam) for $45 \mathrm{~min}$, and the reaction products were visualized by immersing the sections in $0.03 \%$ diaminobenzidine solution containing $2 \mathrm{mM}$ hydrogen peroxide for 1-5 min. Nuclei were lightly stained with Mayer's hematoxylin. The bound antibodies were detected with a biotin-streptavidin-peroxidase system (Vector Laboratories, Inc., Burlingame, CA, USA), and 3,3'-diaminobenzidine hydrochloride (Sigma-Aldrich, St. Louis, MO, USA) was used as the chromogen. Two independent pathologists from the Department of Pathology, Yangpu Hospital, Tongji University School of Medicine, performed the quantitative assessment of immunohistochemical staining. Staining in nuclei was graded as: 0 , no evidence of immunoreactive cells; 1 , the proportion of immunoreactive cells is $\langle 20 \%$; 2 , the proportion of immunoreactive cells is $20-70 \%$; or 3 , the proportion of immunoreactive cells is $>70 \%$. Immunohistochemical reactivity was evaluated and classified into 4 groups: i) Negative (-); ii) weakly positive $(+)$; iii) moderately positive $(++)$; or iv) strongly positive $(+++)$.

Statistics. Data are presented as the mean \pm standard deviation. Statistical tests were performed using SPSS 17.0 (SPSS, Inc., Chicago, IL, USA). The Student's $t$-test (two-tailed) was used make comparisons between two groups, the correlation between HOXD10 and RhoC was assessed using Spearman's rank correlation coefficient and differences between two groups for immunohistochemistry were analyzed using the Mann-Whitney $\mathrm{U}$ test. $\mathrm{P}<0.05$ were considered to indicate a statistically significant difference.

\section{Results}

Patient characteristics. A total of 126 patients with CRC, who had not previously received preoperative radiotherapy or 
Table I. Patient characteristics $(n=126)$.

\begin{tabular}{|c|c|c|}
\hline Characteristic & $\begin{array}{c}\text { No. of } \\
\text { patients }(\%)\end{array}$ & P-value \\
\hline Tumor site & & 0.043 \\
\hline Colon & $74(58.7)$ & \\
\hline Rectum & $52(41.3)$ & \\
\hline Liver metastases & $3(2.4)$ & \\
\hline Multifocal & $1(0.7)$ & \\
\hline Age, years & & 0.763 \\
\hline$\geq 50$ & $64(50.8)$ & \\
\hline$<50$ & $62(49.2)$ & \\
\hline Size of tumor & & 0.013 \\
\hline$\leq 5 \mathrm{~cm}$ diameter & $80(63.5)$ & \\
\hline$>5 \mathrm{~cm}$ diameter & $46(36.5)$ & \\
\hline Gender & & 0.435 \\
\hline Male & $74(58.7)$ & \\
\hline Female & $52(41.3)$ & \\
\hline Tumor classification & & 0.023 \\
\hline $\mathrm{T} 1$ & $9(7.1)$ & \\
\hline $\mathrm{T} 2$ & $31(24.6)$ & \\
\hline $\mathrm{T} 3$ & $81(64.3)$ & \\
\hline $\mathrm{T} 4$ & $5(4.0)$ & \\
\hline Lymph node status & & 0.016 \\
\hline N0 & $66(52.4)$ & \\
\hline N1 & $42(33.3)$ & \\
\hline $\mathrm{N} 2$ & $18(14.3)$ & \\
\hline Histological differentiation & & 0.018 \\
\hline Well & $49(38.9)$ & \\
\hline Moderate & $69(54.8)$ & \\
\hline Poor & $8(6.3)$ & \\
\hline Stage & & 0.007 \\
\hline $\mathrm{I}$ & $19(15.1)$ & \\
\hline II & $45(35.7)$ & \\
\hline III & $62(49.2)$ & \\
\hline HOXD10 staining & & 0.025 \\
\hline Strong $(>1+)$ & $43(34.1)$ & \\
\hline Weak $(\leq 1+)$ & $83(65.9)$ & \\
\hline RhoC staining & & 0.014 \\
\hline Strong $(>1+)$ & 75 (59.5) & \\
\hline Weak $(\leq 1+)$ & $51(40.5)$ & \\
\hline
\end{tabular}

The model was used to analyze the effect of associated factors on miR-10b levels in CRC patients. $\mathrm{P}<0.05$ was considered statistically significant.

chemotherapy, were enrolled in the present study. All patients underwent surgical resection at the Department of General Surgery, Yangpu Hospital, Shanghai Tongji University School of Medicine. All postoperative samples were confirmed as CRC through histopathological evaluation. The study consisted of 74 male and 52 female patients, of which 46 patients possessed tumors that were $>5 \mathrm{~cm}$ in diameter and 80 possessed tumors of $\leq 5 \mathrm{~cm}$ in diameter. Good, moderate and poor tumor differentiation was detected in 49, 69 and 8 cases, respectively. In total, 42 patients exhibited N1 lymph node metastasis and 18 patients exhibited N2 lymph node metastasis. A total of 19 cases were in stage I, 45 cases were in stage II and 62 cases were in stage III of disease. The characteristics of the enrolled patients are listed in Table I.

miR-10b is highly expressed in primary CRC samples. In order to explore the potential role of miR-10b in CRC development, the expression of miR-10b was investigated in tumor specimens obtained from $126 \mathrm{CRC}$ patients. The patients were classified into two groups, according to the presence or absence of lymph node metastasis; significant differences were identified in the mean expression levels of miR-10b and HOXD10 between the two groups $(\mathrm{P}=0.001$ and $\mathrm{P}=0.005)$. The results of the $\mathrm{RT}-\mathrm{qPCR}$ indicated that the mean expression level of miR-10b in tumor tissues with positive lymph node metastasis was 1.8 -fold greater compared with tumor tissues without lymph node metastasis. In addition, the mean expression level of HOXD10 in tumor tissues with positive lymph node metastasis was 0.58 -fold greater compared with tumor tissue without lymph node metastasis (Fig. 1A). Significant differences in the mean miR-10b expression level were also indicated between the CRC tissue group and the healthy individuals and adjacent tissues $(\mathrm{P}=0.012$ and $\mathrm{P}=0.001$; Fig. 1B), while there was no statistically significant difference between the adjacent non-tumor tissue group and the normal control tissue group $(\mathrm{P}=0.986)$. There was no significant difference in the expression levels of miR-10b with respect to age and gender $(\mathrm{P}=0.156)$. The results showed that there were significant differences in the expression of miR-10b in cases of $\mathrm{CRC}$ with varying tumor diameter and histological differentiation $(\mathrm{P}=0.013$ and $\mathrm{P}=0.018)$. A significant difference in miR-10b expression was also indicated between stage I+II and stage III tumors $(\mathrm{P}=0.007$; Fig. $1 \mathrm{C})$. There were significant differences in miR-10b expression between cases with liver metastasis and those with multifocal metastases $(\mathrm{P}=0.004)$. A statistically significant difference was identified between the level of miR-10b and HOXD10 expression ( $\mathrm{P}=0.009$; Fig. 1D). These data suggest that miR-10b may be involved in the process of $\mathrm{CRC}$ metastasis. In addition, an inverse correlation was identified between miR-10b and HOXD10 expression.

Western blot analysis of protein expression of HOXD10 in CRC samples. Since the decreased expression of HOXD10 protein has been shown to enhance the invasiveness and metastasis of tumors (20), the present study aimed to determine whether miR-10b would inhibit the translation of HOXD10 mRNA, thereby affecting the expression of downstream targets of RhoC. As shown in Fig. 2, there were significant differences in the mean level of HOXD10 expression between the CRC tissue group and the adjacent non-tumor or normal control tissue group ( $\mathrm{P}=0.001$; Fig. $2 \mathrm{~A})$, while there was no statistical significance between the adjacent non-tumor tissue group and the normal control tissue group $(\mathrm{P}=0.490)$. RhoC protein levels were dramatically upregulated in tumor tissues with positive lymph node metastasis compared with tissues with no lymph node metastasis $(\mathrm{P}<0.01)$. There were also significant differences in HOXD10 protein level between the CRC samples with positive lymph node metastasis and the CRC samples 
A Relative level of pcr in colorectal cancer tissue

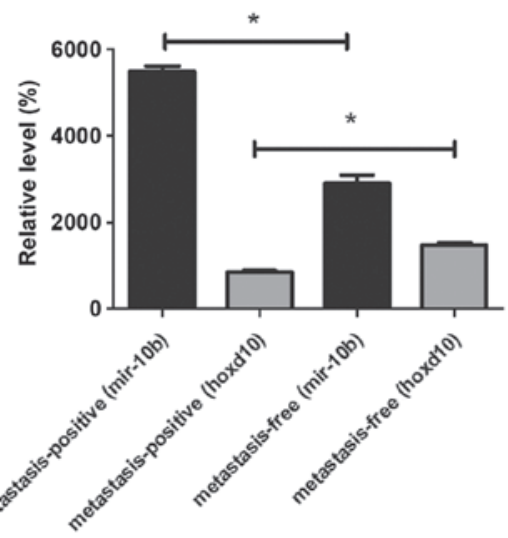

B

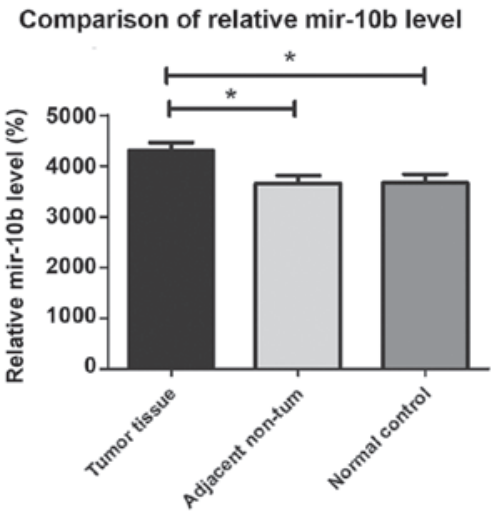

C

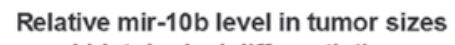
and histological differentiations

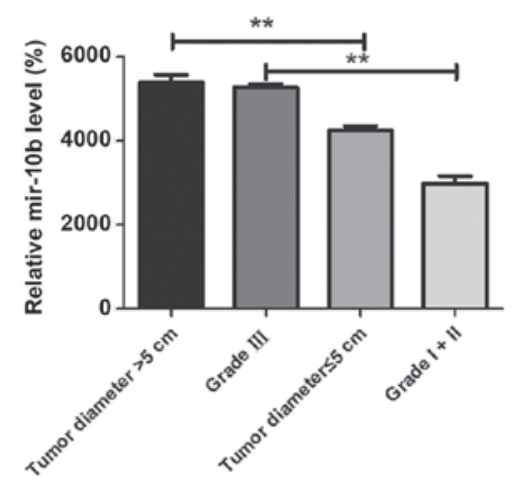

D Relative level of pcr in colorectal cancer tissue

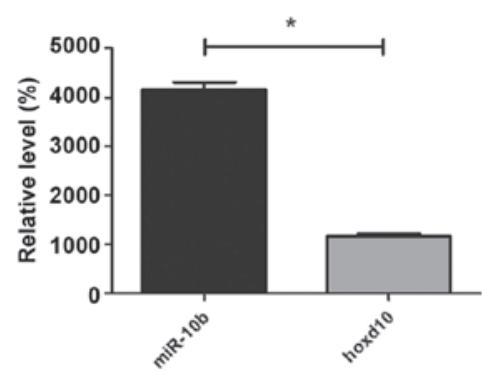

Figure 1. miR-10b expression in various tissues. (A) Comparison of miR-10b and HOXD10 expression between the samples with positive and negative lymph node metastasis in the CRC tissue group. (B) Amplification with $\Delta \Delta \mathrm{Cq}$ values of mir-10b in the $\mathrm{CRC}$, adjacent non-tumor and normal control tissue groups. (C) Relative miR-10b expression in CRC samples with various tumor diameter and histological differentiations. (D) Comparison of miR-10b and HOXD10 expression in the CRC tissue group $\left({ }^{*} \mathrm{P}<0.01 ;{ }^{* *} \mathrm{P}<0.05\right)$. miR-10b, microRNA-10b; HOXD10, homeobox D10; CRC, colorectal cancer.
A Comparison of relative hoxd10 protein level of wb

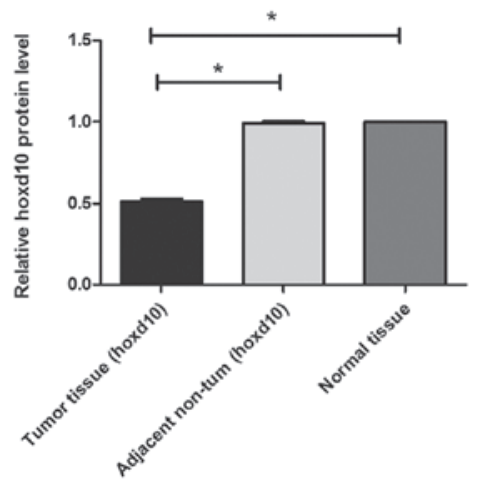

B Comparison of hoxd10 and rhoc protein level of wb

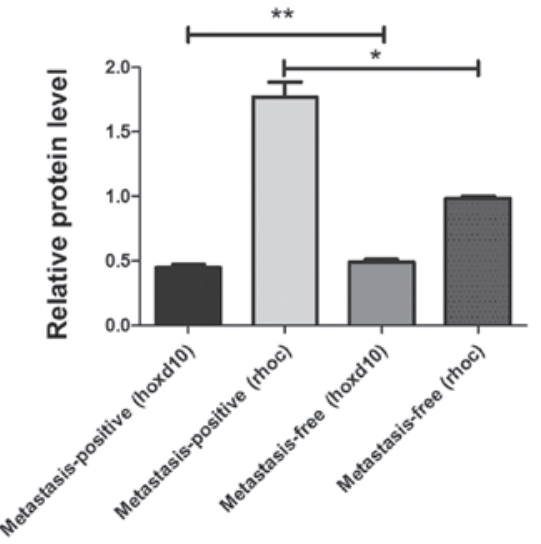

C Correlation between hoxd10 and rhoc protein in crc

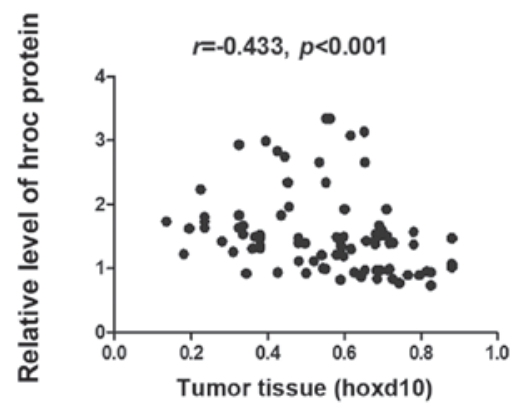

D Correlation between hoxd10 and rhoc protein in crc

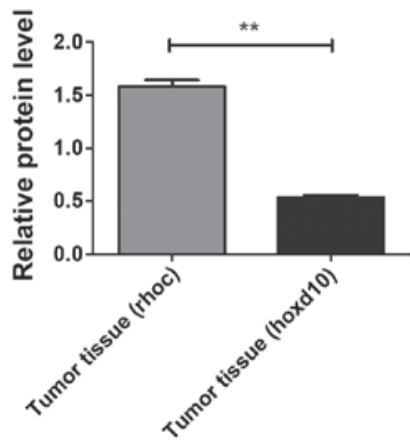

Figure 2. Correlation between protein expression of RhoC and HOXD10 in the CRC tissue group. (A) Protein expression of HOXD10 in the CRC, adjacent non-tumor and normal control tissue groups. (B) Comparison of RhoC and HOXD10 protein expressions in CRC samples with or without positive lymph node metastasis. (C) Inverse correlation between HOXD10 and RhoC protein expression in $\mathrm{CRC}$ samples $(r=-0.433$; $\mathrm{P}<0.001)$. (D) Comparison of RhoC and HOXD10 expression in the western blot analysis in the CRC tissue group $\left({ }^{*} \mathrm{P}<0.01 ;{ }^{* *} \mathrm{P}<0.05\right)$. RhoC, ras homolog family member $\mathrm{C}$; HOXD10, homeobox D10; CRC, colorectal cancer. 


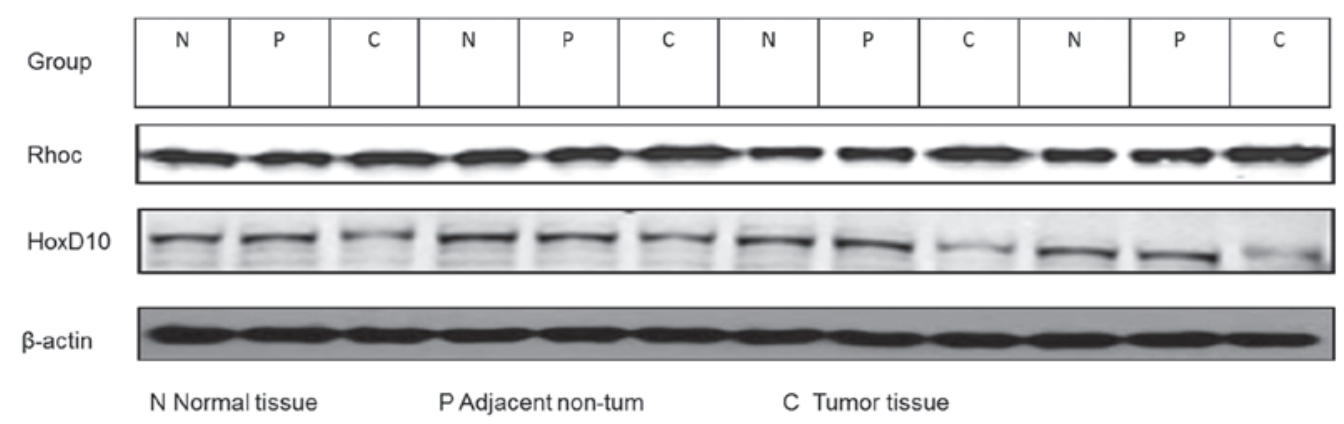

Figure 3. Protein expression of RhoC and HOXD10 in the CRC group. HOXD10 and RhoC were detected by western blot analysis. An inverse correlation between HOXD10 and RhoC protein expression was found. $\beta$-actin was used as an internal reference. $\mathrm{N}$, normal tissue; $\mathrm{P}$, adjacent non-tumor tissues; $\mathrm{C}$, tumor tissues; RhoC, ras homolog family member C; HOXD1, homeobox D1.

A
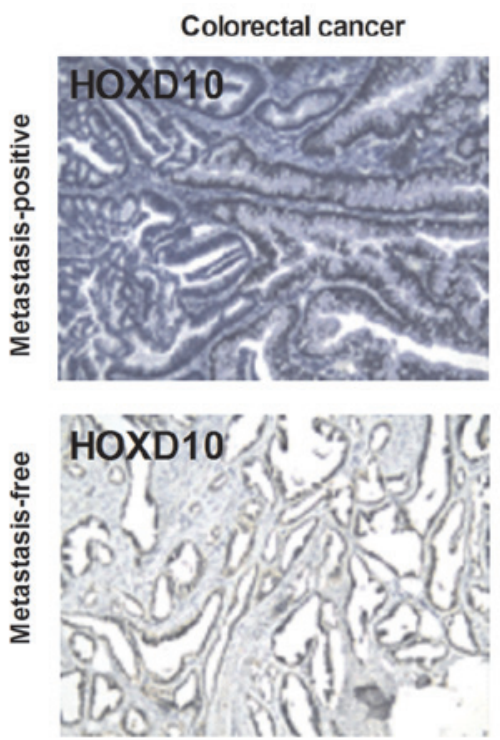

control
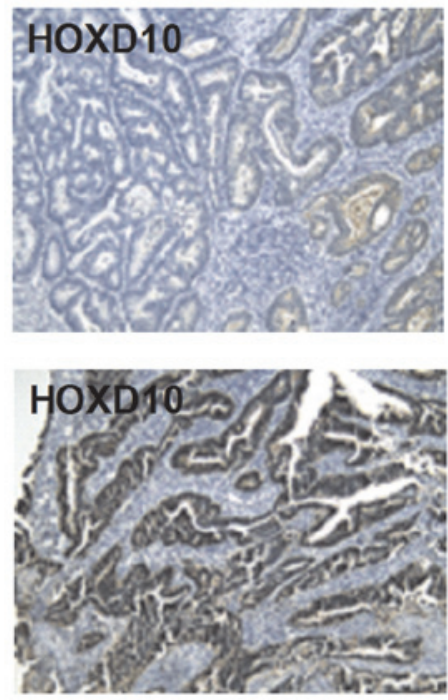

B

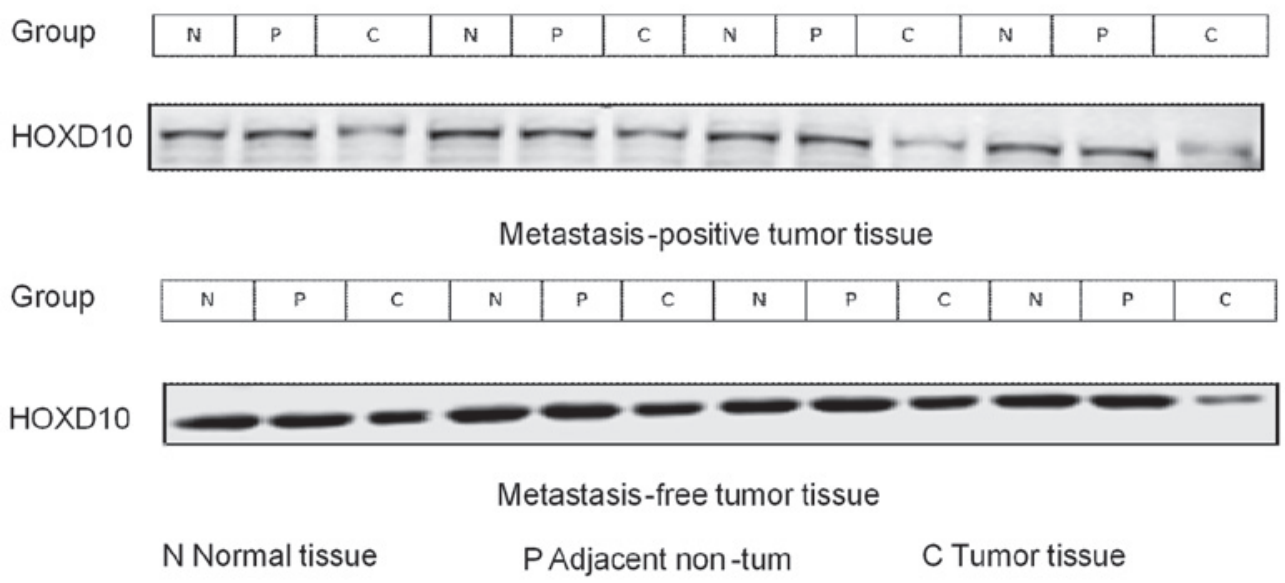

Figure 4. Association between HOXD10 expression and CRC metastasis. (A) Comparison of HOXD10 expression by immunohistochemistry between the samples with positive and negative lymph node metastasis in the CRC tissue group. (B) Comparison of HOXD10 expression by western blot analysis between the samples with positive and negative lymph node metastasis in the CRC tissue group. $\beta$-actin was used as an internal reference. CRC invasion and metastasis were enhanced by downregulated HOXD10. N, normal tissue; P, adjacent non-tumor tissues; C, tumor tissues; HOXD10, homeobox D1.

without positive lymph node metastasis ( $\mathrm{P}=0.014)$ (Fig. 2B). As shown in Figs. 2C and 3, there was a significant inverse correlation between HOXD10 protein level and protein level $(r=-0.433 ; \mathrm{P}<0.001)$. There was statistically significant difference between RhoC protein level and HOXD10 protein level in the $\mathrm{CRC}$ tissue group ( $\mathrm{P}=0.002$; Fig. 2D).
Difference in protein expression of HOXD10 between metastasis-positive and metastasis-free samples. The results of the western blot analysis indicated that HOXD10 expression in tumor tissues from the metastasis-positive group were decreased compared with in metastasis-free tissues (Fig. 2B). To confirm the result of western blot analysis, 
immunohistochemistry was also performed to detect the expression of HOXD10. HOXD10 immunoexpression was found to be attenuated in metastasis-positive tissues compared with metastasis-free tissues (Fig. 4A). A significant inverse correlation in the protein expression of HOXD10 was also observed in metastasis-positive tissues compared with metastasis-free tissues (Fig. 4B). These data suggest that HOXD10 may be involved in the process of CRC metastasis.

\section{Discussion}

The HOXD10 gene encodes transcription factors and exerts functions mainly through the activation or inhibition of downstream target genes (21). HOXD10 was previously considered to be a major factor in the negative regulation of tumor metastasis, as the overexpression of miR-10b was hypothesized to increase RhoC and AKT signaling by targeting HOXD10, thus facilitating invasion in tumors (22). The present study measured the expression of miR-10b, HOXD10 and RhoC in healthy control subjects and CRC patients. miR-10b was found to be highly expressed in CRC tissues, accompanied by the increased expression of RhoC. Consistent with previous findings, that $\mathrm{miR}-10 \mathrm{~b}$ could drive tumor metastasis (23-25), the present study found that increased miR-10b also contributed to metastasis in CRC. In addition, increased levels of miR-10b were detected in poorly-differentiated CRC tissues, indicating a strong capability for metastasis. HOXD10 expression was found to be attenuated in lymph node metastasis-positive CRC tissues, compared with metastasis-free CRC. These results suggest that miR-10b could reduce the threshold of metastasis in $\mathrm{CRC}$ as the positive regulator, which was inversely associated to HOXD10.

There were significant differences in the mean level of miR-10b expression between the tumor and the adjacent non-tumor tissues $(\mathrm{P}<0.05)$, while there was no significant difference between the adjacent non-tumor and normal CRC tissue ( $\mathrm{P}>0.05$; Fig. 1). There were significant differences in miR-10b level between CRC cases of various stages, tumor diameter, lymph node metastasis status and differentiation. Furthermore, there was a statistically significant difference between the multi- and mono-focal cases (Table I). miR-10b was previously observed to be abnormally expressed in several tumor types, including glioma, pancreatic adenocarcinoma and glioblastoma (26-28). The current findings, together with the findings of previous studies, suggest the involvement of miR-10b in the metastatic behavior of CRC $(29,30)$.

The present study found significantly greater concentrations of miR-10b in CRC tissues compared with in adjacent non-tumor tissues and normal tissues from healthy controls. The concentrations of miR-10b increased in line with a later clinical stage and lymph node metastasis. In addition, miR-10b expression in high-grade CRC with multifocal lesions and liver metastases was significantly increased compared with CRC patients with a single lesion and those without liver metastasis. These findings indicate that miR-10b may be involved in the invasion and migration of CRC and may provide a novel therapeutic target. In the present study, miR-10b expression levels were elevated in metastasis-positive CRC specimens compared with metastasis-free tumor tissues, and this elevation was accompanied with the downregulation of HOXD10. HOXD10 has been previously found to repress the expression of genes involved in tumor metastasis, including RhoC (12). miR-10b overexpression may increase RhoC expression, indicating that increased RhoC contributed to miR-10b-induced invasiveness, as the target gene of HOXD10. In addition, the expression of miR-10b was greater in poorly-differentiated CRC tissues compared with well-differentiated ones. Spearman's rank correlation coefficient revealed that there was a strong negative correlation between HOXD10 and RhoC protein levels (Fig. 2C). The results of the present study showed that the overexpression of miR-10b increased RhoC expression by targeting HOXD10, thus facilitating the invasion and metastasis of CRC.

In conclusion, the present study suggests that increased miR-10b levels are associated with the degree of metastasis in $\mathrm{CRC}$ patients. The increased expression of RhoC and the downregulation of HOXD10 were correlated with high expression levels of miR-10b. These results may elucidate the underlying mechanisms and provide a novel therapeutic target for inhibiting the invasion and metastasis of CRC.

\section{Acknowledgements}

The present study was supported by grants from the Youth Issues of Shanghai Municipal Health Bureau of China, Shanghai, China (grant no., 20124y155).

\section{References}

1. Dai J, Zou T, Wang L, Zhang Y and Liu Y: Investigation of the interaction between quercetin and human serum albumin by multiple spectra, electrochemical impedance spectra and molecular modeling. Luminescence 29: 1154-1161, 2014.

2. Zhong L, Zhang X and Covasa M: Emerging roles of lactic acid bacteria in protection against colorectal cancer. World J Gastroenterol 20: 7878-7886, 2014.

3. Dueland S, Hagness M, Line PD, Guren TK, Tveit KM and Foss A: Is liver transplantation an option in colorectal cancer patients with nonresectable liver metastases and progression on all lines of standard chemotherapy? Ann Surg Oncol 22: 2195-2200, 2015.

4. Cleven AH, Derks S, Draht MX, Smits KM, Melotte V, Van Neste L, Tournier B, Jooste V, Chapusot C, Weijenberg MP, et al: CHFR promoter methylation indicates poor prognosis in stage II microsatellite stable colorectal cancer. Clin Cancer Res 20: 3261-3271, 2014.

5. Chen P, Xi Q, Wang Q and Wei P: Downregulation of microRNA-100 correlates with tumor progression and poor prognosis in colorectal cancer. Med Oncol 31: 235, 2014.

6. Adams SV, Newcomb PA, Burnett-Hartman AN, Wurscher MA, Mandelson M, Upton MP, Zhu LC, Potter JD and Makar KW: Rare circulating microRNAs as biomarkers of colorectal neoplasia. PLoS One 9: e108668, 2014.

7. Drusco A, Nuovo GJ, Zanesi N, Di Leva G, Pichiorri F, Volinia S, Fernandez C, Antenucci A, Costinean S, Bottoni A, et al: MicroRNA profiles discriminate among colon cancer metastasis. PLoS One 9: e96670, 2014.

8. Liang G, Li J, Sun B, Li S, Lü L, Wang Y, Chen B and Xiao Z: Deep sequencing reveals complex mechanisms of microRNA deregulation in colorectal cancer. Int J Oncol 45: 603-610, 2014.

9. Phua LC, Chue XP, Koh PK, Cheah PY, Chan EC and Ho HK: Global fecal microRNA profiling in the identification of biomarkers for colorectal cancer screening among Asians. Oncol Rep 32: 97-104, 2014.

10. Yin J, Bai Z, Song J, Yang Y, Wang J, Han W, Zhang J, Meng H, Ma X, Yang Y, et al: Differential expression of serum miR-126, miR-141 and miR-21 as novel biomarkers for early detection of liver metastasis in colorectal cancer. Chin J Cancer Res 26: 95-103, 2014 
11. Haque I, Banerjee S, Mehta S, De A, Majumder M, Mayo MS Kambhampati S, Campbell DR and Banerjee SK: Cysteine-rich 61-connective tissue growth factor-nephroblastoma overexpressed 5 (CCN5)/Wnt-1-induced signaling protein-2 (WISP-2) regulates microRNA-10b via hypoxia-inducible factor-1 $\alpha$-TWIST signaling networks in human breast cancer cells. J Biol Chem 286: 43475-43485, 2011.

12. Liu Z, Zhu J, Cao H, Ren H and Fang X: MiR-10b promotes cell invasion through RhoC-AKT signaling pathway by targeting HOXD10 in gastric cancer. Int J Oncol 40: 1553-1560, 2012.

13. Yu X, Li Z, Shen J, Wu WK, Liang J, Weng X and Qiu G: MicroRNA-10b promotes nucleus pulposus cell proliferation through RhoC-Akt pathway by targeting HOXD10 in intervetebral disc degeneration. PLoS One 8: e83080, 2013.

14. Bu Q, Tang HM, Tan J, Hu X and Wang DW: Expression of RhoC and ROCK-1 and their effects on MAPK and Akt proteins in prostate carcinoma. Zhonghua Zhong Liu Za Zhi 33: 202-206, 2011 (In Chinese).

15. Nishida N, Yamashita S, Mimori K, Sudo T, Tanaka F, Shibata K, Yamamoto H, Ishii H, Doki Y and Mori M: MicroRNA-10b is a prognostic indicator in colorectal cancer and confers resistance to the chemotherapeutic agent 5-fluorouracil in colorectal cancer cells. Ann Surg Oncol 19: 3065-3071, 2012

16. Wang YF, Li Z, Zhao XH, Zuo XM, Zhang Y, Xiao YH, Li J and Peng ZH: MicroRNA-10b is upregulated and has an invasive role in colorectal cancer through enhanced Rhoc expression. Oncol Rep 33: $1275-1283,2015$.

17. von Winterfeld M, Hoffmeister M, Ingold-Heppner B, Jansen L Tao S, Herpel E, Schirmacher P, Dietel M, Chang-Claude J, Autschbach F, et al: Frequency of therapy-relevant staging shifts in colorectal cancer through the introduction of pN1c in the 7th TNM edition. Eur J Cancer 50: 2958-2965, 2014.

18. Rickham PP: Human experimentation. Code of ethics of the World Medical Association. Declaration of Helsinki. Br Med J 2: 177, 1964.

19. Wang L, Chen S, Xue M, Zhong J, Wang X, Gan L, Lam EK, Liu X, Zhang J, Zhou T, et al: Homeobox D10 gene, a candidate tumor suppressor, is downregulated through promoter hypermethylation and associated with gastric carcinogenesis. Mol Med 18: 389-400, 2012.

20. Xiao H, Li H, Yu G, Xiao W, Hu J, Tang K, Zeng J, He W, Zeng G, $\mathrm{Ye} \mathrm{Z}$ and $\mathrm{Xu} \mathrm{H}$ : MicroRNA-10b promotes migration and invasion through KLF4 and HOXD10 in human bladder cancer. Oncol Rep 31: 1832-1838, 2014
21. Xue M, Fang Y, Sun G, Zhuo W, Zhong J, Qian C, Wang L, Wang L, Si J and Chen S: IGFBP3, a transcriptional target of homeobox D10, is correlated with the prognosis of gastric cancer. PLoS One 8: e81423, 2013.

22. Chen W, Cai F, Zhang B, Barekati Z and Zhong XY: The level of circulating miRNA-10b and miRNA-373 in detecting lymph node metastasis of breast cancer: Potential biomarkers. Tumour Biol 34: 455-462, 2013.

23. Severino $\mathrm{P}$, Brüggemann $\mathrm{H}$, Andreghetto FM, Camps $\mathrm{C}$, Klingbeil Mde F, de Pereira WO, Soares RM, Moyses R, Wünsch-Filho V, Mathor MB, et al: MicroRNA expression profile in head and neck cancer: HOX-cluster embedded microRNA-196a and microRNA-10b dysregulation implicated in cell proliferation. BMC Cancer 13: 533, 2013.

24. Yigit MV, Ghosh SK, Kumar M, Petkova V, Kavishwar A Moore A and Medarova Z: Context-dependent differences in miR-10b breast oncogenesis can be targeted for the prevention and arrest of lymph node metastasis. Oncogene 32: 1530-1538, 2013.

25. Guessous F, Alvarado-Velez M, Marcinkiewicz L, Zhang Y, Kim J, Heister S, Kefas B, Godlewski J, Schiff D, Purow B and Abounader R: Oncogenic effects of miR-10b in glioblastoma stem cells. J Neurooncol 112: 153-163, 2013.

26. Karsy M, Arslan E and Moy F: Current progress on understanding MicroRNAs in glioblastoma multiforme. Genes Cancer 3: 3-15, 2012.

27. Xue Y, Abou Tayoun AN, Abo KM, Pipas JM, Gordon SR, Gardner TB, Barth RJ Jr, Suriawinata AA and Tsongalis GJ: MicroRNAs as diagnostic markers for pancreatic ductal adenocarcinoma and its precursor, pancreatic intraepithelial neoplasm. Cancer Genet 206: 217-221, 2013.

28. Moghimi-Dehkordi B and Safaee A: An overview of colorectal cancer survival rates and prognosis in Asia. World J Gastrointest Oncol 4: 71-75, 2012.

29. Sarkar S, Mukherjee R, Paira SK, Roy B, Banerjee S and Mukherjee SK: Profile of colorectal cancer in Eastern India. J Indian Med Assoc 110: 901-903, 2012.

30. Wong MC, Lam TY, Tsoi KK, Chan VC, Hirai HW, Ching JY and Sung JJ: Predictors of advanced colorectal neoplasia for colorectal cancer screening. Am J Prev Med 46: 433-439, 2014. 